\title{
Stabilization of strictly dissipative discrete time systems with discounted optimal control*
}

\author{
Vladimir Gaitsgory \\ Department of Mathematics \\ Macquarie University \\ NSW 2109, Australia \\ vladimir.gaitsgory@mq.edu.au \\ Lars Grüne, Matthias Höger ${ }^{\dagger}$ \\ Mathematisches Institut \\ Universität Bayreuth \\ 95440 Bayreuth, Germany \\ lars.gruene, matthias.hoeger@uni-bayreuth.de \\ Christopher M. Kellett, Steven R. Weller \\ School of Electrical Engineering and Computing \\ University of Newcastle \\ Callaghan, NSW 2308, Australia \\ chris.kellett, steven.weller@newcastle.edu.au
}

August 17, 2017

\begin{abstract}
We consider stabilization of an equilibrium point via infinite horizon discounted optimal control in discrete-time. In addition to applications in economics and social sciences, discounted optimal control is a commonly used numerical technique guaranteeing solvability of certain classes of optimal control problems. In this paper, we present conditions based on strict dissipativity that ensure that the optimally controlled system is asymptotically stable or practically asymptotically stable. These conditions are shown to be complementary to recently proposed conditions based on a detectability property. Illustrative examples are provided.
\end{abstract}

Keywords: Stabilization, Discounted optimal control, Strict Dissipativity, Lyapunov function

* Parts of the research for this paper was carried out while Lars Grüne was visiting the University of Newcastle and Macquarie University, Sydney, Australia. The research was supported by the Australian Research Council (ARC) Discovery Grants DP130104432, DP120100532, and DP160102138 and by the Deutsche Forschungsgemeinschaft Grant GR1569/13-1.

${ }^{\dagger}$ Matthias Höger is also affiliated with Siemens AG, Division Power and Gas, 91058 Erlangen, Germany, matthias.hoeger@siemens.com 


\section{Introduction}

Asymptotic stabilization of an equilibrium point via optimal control techniques has long been used as a method for computing feedback stabilizers, particularly in the context of the infinite-horizon linear quadratic regulator problem [21] (cf. [2, Sections 9.2.3, 9.2.6]). In this context, under appropriate assumptions, the solution to the algebraic Riccati equation provides a static state feedback stabilizer that also solves the optimal control problem.

In the more general case of nonlinear systems with positive definite (possibly nonquadratic) stage costs, the value function for a particular optimal control problem is still known to be a control Lyapunov function $[32,14,22]$. Moreover, in the discrete time setting considered in this paper, deriving a feedback stabilizer from a control Lyapunov function is relatively straightforward. However, solving the optimal control problems posed in $[14,22]$ is numerically difficult.

One possible solution to the numerical difficulty of computing closed-loop optimal controls is via receding horizon or model predictive control (MPC) [11]. Indeed, reference [11] shows that as the horizon length grows, the solutions obtained via receding horizon control closely approximate the infinite horizon solution.

A somewhat similar solution to this numerical difficulty arises by considering an infinite horizon optimal control problem with a discounted cost. Here, the stage cost at the current time carries a greater weighting than the stage cost at future times. Consequently, states and controls far into the future have a limited effect on the present, suggesting the possibility of truncating the cost function once the discounted stage cost becomes sufficiently small, not unlike taking a sufficiently large horizon in the context of receding horizon control. Moreover, for discounted optimal control problems the dynamic programming operator has a certain contraction property which again simplifies the numerical solution [3].

In addition to their potential as a numerical technique, optimal control problems with a discounted stage cost commonly arise in economic applications [30, 29] and in social sciences [7]. In the context of welfare maximization problems involving rational decision-makers, consumption at the current time provides greater welfare than consumption in the future, with the discount factor reflecting a trade-off between current and future consumption $[17,18,25]$.

For discounted optimal control problems for nonlinear discrete time systems, sufficient conditions for (practical) asymptotic stability of the optimally controlled system were first given in [27] (see also [26]), based on a nonlinear detectability condition introduced in [8] in the context of MPC. In this paper we follow a different path and use strict dissipativity, which was also recently studied in the context of $\mathrm{MPC}[1,5,11]$ and was extended to the discounted setting in [12]. As we will illustrate by an example in Section 5, this approach applies to a different class of systems than the detectability based analysis. We present both conditions for semiglobal practical asymptotic stability for discount factor $\beta$ sufficiently close to 1 , and conditions for asymptotic stability for fixed (and possibly rather small) discount factors $\beta \in(0,1)$. Some of these conditions can be seen as discrete time analogues of continuous time results in [6], but the dissipativity based analysis is to the best of our knowledge novel both in discrete and in continuous time. 
The paper is organized as follows. In Section 2 we provide the necessary problem setup and definitions, and in Section 3 we provide a sufficient condition for practical asymptotic stability using discounted optimal controls for fixed discount factor $\beta \in(0,1)$. In Section 4 we extend these results to strictly dissipative optimal control problems and in Section 5 we compare our dissipativity based approach with the detectability based approach from [27]. Section 6 discusses two alternative conditions for non-practical asymptotic stability and illustrates the conservativeness of some of our conditions by means of an example. Some brief conclusions are provided in Section 7 .

\section{Problem formulation}

Let $X$ and $U$ be normed spaces. Given a discount factor $0<\beta<1$ and a stage cost $g: X \times U \rightarrow \mathbb{R}$ we consider the discounted optimal control problem

$$
\text { minimize } J_{\beta}\left(x_{0}, u(\cdot)\right)=\sum_{k=0}^{\infty} \beta^{k} g(x(k), u(k))
$$

with respect to the control functions $u(\cdot) \in \mathcal{U}=\left\{u: \mathbb{N}_{0} \rightarrow U\right\}$, where $\mathbb{N}_{0}$ denotes the natural numbers including 0 . The state trajectory $x(k)$ is given by the discrete time control system

$$
x(k+1)=f(x(k), u(k)), \quad k \in \mathbb{N}_{0}
$$

and the minimization is subject to the initial condition $x(0)=x_{0}$ and the control and state constraints $u(t) \in \mathbb{U}, x(t) \in \mathbb{X}$, where $\mathbb{X}$ and $\mathbb{U}$ are subsets of $X$ and $U$, respectively. The functions $f: X \times U \rightarrow X$ and $g: X \times U \rightarrow \mathbb{R}$ are assumed to be continuous. We assume that the set $\mathbb{X}$ is viable, i.e., for any $x_{0} \in \mathbb{X}$ there exists at least one $u(\cdot) \in \mathcal{U}$ with $u(k) \in \mathbb{U}$ and $x(k) \in \mathbb{X}$ for all $k \in \mathbb{N}_{0}$. Control functions with this property will be called admissible and the set of all admissible control functions will be denoted by $\mathcal{U}_{a d}$. The fact that we impose the state constraints when solving (2.1) implies that the minimization in (2.1) is carried out over the set of admissible control functions only.

We define the optimal value function of the problem as

$$
V_{\beta}\left(x_{0}\right):=\inf _{u(\cdot) \in \mathcal{U}_{a d}} J_{\beta}\left(x_{0}, u(\cdot)\right) .
$$

Throughout the paper we assume that $V_{\beta}\left(x_{0}\right)$ is finite for all $x_{0} \in \mathbb{X}$. An admissible control function $u^{*}(\cdot) \in \mathcal{U}_{a d}$ is called optimal for initial condition $x_{0} \in \mathbb{X}$ if the identity

$$
J_{\beta}\left(x_{0}, u^{*}(\cdot)\right)=V_{\beta}\left(x_{0}\right)
$$

holds. We summarize a few statements on optimal value functions and optimal controls which can be found, e.g., in [15, Chapter 4]. The optimal value function satisfies the dynamic programming principle

$$
V_{\beta}(x)=\inf _{u \in \mathbb{U}: f(x, u) \in \mathbb{X}}\left\{g(x, u)+\beta V_{\beta}(f(x, u))\right\} .
$$

If $u^{*}(\cdot)$ is an optimal control sequence for initial condition $x_{0}=x \in \mathbb{X}$, then the identity

$$
V_{\beta}(x)=g\left(x, u^{*}(0)\right)+\beta V_{\beta}\left(f\left(x, u^{*}(0)\right)\right)
$$


holds. In this case, the "inf" in (2.3) is actually a "min". If this holds for all $x \in \mathbb{X}$, we can define a (not necessarily unique) map $\mu^{*}: \mathbb{X} \rightarrow \mathbb{U}$ which assigns a minimizer of the right hand side of $(2.3)$ to each $x$, i.e.,

$$
\mu^{*}(x) \in \underset{u \in \mathbb{U}: f(x, u) \in \mathbb{X}}{\operatorname{argmin}}\left\{g(x, u)+\beta V_{\beta}(f(x, u))\right\} .
$$

Then, any such map $\mu^{*}$ is an optimal feedback law, i.e., the closed-loop trajectories defined by

$$
x^{*}(0)=x_{0}, \quad x^{*}(k+1)=f\left(x^{*}(k), \mu^{*}\left(x^{*}(k)\right)\right), \quad k \in \mathbb{N}_{0}
$$

are optimal trajectories and $u^{*}(k)=\mu^{*}\left(x^{*}(k)\right)$ is an optimal control sequence for initial value $x_{0}$. In what follows, we refer to (2.6) as the optimal closed-loop.

Our goal in this paper is to derive conditions under which optimal feedback laws $\mu^{*}$ asymptotically stabilize a desired equilibrium point for the closed-loop system. To this end, we use the following definitions.

- We say that a pair $\left(x^{e}, u^{e}\right) \in \mathbb{X} \times \mathbb{U}$ is an equilibrium if $f\left(x^{e}, u^{e}\right)=x^{e}$.

- An equilibrium is called asymptotically stable, if there exists a function ${ }^{1} \eta \in \mathcal{K} \mathcal{L}$ such that all closed-loop trajectories $x(k)$ satisfy the inequality

$$
\left\|x(k)-x^{e}\right\| \leq \eta\left(\left\|x(0)-x^{e}\right\|, k\right)
$$

for all $k \in \mathbb{N}_{0}$.

- We say that a set $P \subset \mathbb{X}$ is forward invariant for the closed-loop, if for all closed-loop trajectories and all $k \in \mathbb{N}$ the implication $x(k) \in P \Rightarrow x(k+1) \in P$ holds.

- For two forward invariant sets $P \subset Y \subset \mathbb{X}$, an equilibrium $\left(x^{e}, u^{e}\right)$ with $x^{e} \in P$ is called $P$-practically asymptotically stable on $Y$, if there exists a function $\eta \in \mathcal{K} \mathcal{L}$ such that all closed-loop trajectories $x(k)$ with $x(0) \in Y$ satisfy the inequality

$$
\left\|x(k)-x^{e}\right\| \leq \eta\left(\left\|x(0)-x^{e}\right\|, k\right)
$$

for all $k \in \mathbb{N}_{0}$ with $x(k) \notin P$.

- For two numbers $\Delta>\delta>0$, an equilibrium $\left(x^{e}, u^{e}\right)$ is called $(\delta, \Delta)$-practically asymptotically stable, if there exists a function $\eta \in \mathcal{K} \mathcal{L}$ such that all closed-loop trajectories $x(k)$ with $\left\|x(0)-x^{e}\right\| \leq \Delta$ satisfy the inequality

$$
\left\|x(k)-x^{e}\right\| \leq \max \left\{\eta\left(\left\|x(0)-x^{e}\right\|, k\right), \delta\right\}
$$

for all $k \in \mathbb{N}_{0}$.

It is easily seen that $P$-practical asymptotic stability on $Y$ implies $(\delta, \Delta)$-practical asymptotic stability if $P \subset B_{\delta}\left(x^{e}\right)$ and $Y \supset \bar{B}_{\Delta}\left(x^{e}\right) \cap \mathbb{X}$. If $Y=\mathbb{X}$ and $P=\left\{x^{e}\right\}$, then $P$-practical asymptotic stability is equivalent to non-practical asymptotic stability.

\footnotetext{
${ }^{1}$ As usual, we say that $\gamma: \mathbb{R}_{0}^{+} \rightarrow \mathbb{R}_{0}^{+}$is a $\mathcal{K}$-function if it is continuous and strictly increasing with $\gamma(0)=0$. It is called a $\mathcal{K}_{\infty}$-function if additionally it is unbounded. A function $\eta: \mathbb{R}_{0}^{+} \times \mathbb{R}_{0}^{+} \rightarrow \mathbb{R}_{0}^{+}$is a $\mathcal{K} \mathcal{L}$-function if it is continuous, for each $t \geq 0$ the map $r \mapsto \eta(r, t)$ is a $\mathcal{K}$-function, and for each $r \geq 0$ the map $t \mapsto \eta(r, t)$ is strictly decreasing and converges to 0 as $t \rightarrow \infty$. By convention, $\eta(0, t)=0$ for all $t \geq 0$.
} 


\section{A condition on the optimal value function}

Theorem 3.1: Let $x^{e} \in \mathbb{X}$ be an equilibrium and consider a discounted optimal control problem with positive definite stage cost w.r.t. $x^{e}$, i.e., there is $\alpha_{1} \in \mathcal{K}_{\infty}$ with

$$
g(x, u) \geq \alpha_{1}\left(\left\|x-x^{e}\right\|\right) \quad \text { for all } x \in \mathbb{X} \text { and } u \in \mathbb{U}
$$

Assume that there is $\alpha_{2} \in \mathcal{K}_{\infty}$ such that the optimal value function $V_{\beta}$ satisfies

$$
V_{\beta}(x) \leq \alpha_{2}\left(\left\|x-x^{e}\right\|\right) \quad \text { for all } x \in \mathbb{X}
$$

and constants $0 \leq \vartheta<\Theta$ and $C \geq 1$ with

$$
V_{\beta}(x) \leq C \inf _{u \in \mathbb{U}} g(x, u) \quad \text { for all } x \in \mathbb{X} \text { with } \vartheta \leq\left\|x-x^{e}\right\| \leq \Theta
$$

and

$$
C<1 /(1-\beta)
$$

Then, whenever $\alpha_{1}(\Theta)>\alpha_{2}(\vartheta) / \beta$, there are forward invariant sets $P \subset Y \subset \mathbb{X}$ with $P \subset$ $\bar{B}_{\delta}\left(x^{e}\right), \delta=\alpha_{1}^{-1}\left(\alpha_{2}(\vartheta) / \beta\right), Y \supset B_{\Delta}\left(x^{e}\right) \cap \mathbb{X}, \Delta=\alpha_{2}^{-1}\left(\alpha_{1}(\Theta)\right)$, such that the equilibrium $\left(x^{e}, u^{e}\right)$ is $P$-practically asymptotically stable on $Y$ for the optimally controlled system. Particularly, the optimal closed-loop system is $(\delta, \Delta)$-practically asymptotically stable. If (3.3) holds for all $x \in \mathbb{X}$, then the equlibrium is asymptotically stable for the optimally controlled system.

Proof. We first observe that, under the assumptions, $V_{\beta}$ satisfies the inequality

$$
V_{\beta}\left(x_{0}\right)=\inf _{u \in \mathcal{U}_{a d}} \sum_{k=0}^{\infty} \beta^{k} g(x(k), u(k)) \geq \inf _{u \in \mathbb{U}} g\left(x_{0}, u\right) \geq \alpha_{1}\left(\left\|x_{0}-x^{e}\right\|\right)
$$

Note that the first inequality in (3.5) implies that $C$ in (3.3) must satisfy $C \geq 1$. In what follows, $x^{*}(\cdot)$ denotes an arbitrary optimal trajectory with corresponding optimal control sequence $u^{*}(\cdot)$. For any such trajectory, (2.4) yields the equation

$$
V_{\beta}\left(x^{*}(k)\right)=g\left(x^{*}(k), u^{*}(k)\right)+\beta V_{\beta}\left(x^{*}(k+1)\right) .
$$

We define the sets

$$
P:=\left\{x \in \mathbb{X} \mid V_{\beta}(x) \leq \alpha_{2}(\vartheta) / \beta\right\} \quad \text { and } \quad Y:=\left\{x \in \mathbb{X} \mid V_{\beta}(x) \leq \alpha_{1}(\Theta)\right\}
$$

These definitions imply $\left\|x-x^{e}\right\| \leq \Theta$ for all $x \in Y$ and $\left\|x-x^{e}\right\|>\vartheta$ for all $x \notin P$, as well as $P \subset \bar{B}_{\delta}\left(x^{e}\right)$ and $Y \subset B_{\Delta}\left(x^{e}\right) \cap \mathbb{X}$ with $\delta$ and $\Delta$ defined in the statement of the theorem. Moreover, the condition $\alpha_{1}(\Theta)>\alpha_{2}(\vartheta) / \beta$ implies $P \subset Y$. 
We first show an auxiliary inequality. Consider an optimal trajectory $x^{*}(\cdot)$ and $k \in \mathbb{N}_{0}$ with $\vartheta \leq\left\|x^{*}(k)-x^{e}\right\| \leq \Theta$. Then, using (3.6), (3.3) and (3.4) we obtain

$$
\begin{aligned}
V_{\beta}\left(x^{*}(k+1)\right)-V_{\beta}\left(x^{*}(k)\right) & =\frac{1}{\beta}\left(\beta V_{\beta}\left(x^{*}(k+1)\right)-\beta V_{\beta}\left(x^{*}(k)\right)\right) \\
& =\frac{1}{\beta}\left(\beta V_{\beta}\left(x^{*}(k+1)\right)-V_{\beta}\left(x^{*}(k)\right)+(1-\beta) V_{\beta}\left(x^{*}(k)\right)\right) \\
& =\frac{1}{\beta}\left(-g\left(x^{*}(k), u^{*}(k)\right)+(1-\beta) V_{\beta}\left(x^{*}(k)\right)\right) \\
& \leq \frac{1}{\beta}\left(-\frac{1}{C} V_{\beta}\left(x^{*}(k)\right)+(1-\beta) V_{\beta}\left(x^{*}(k)\right)\right) \\
& =\frac{\kappa}{\beta} V_{\beta}\left(x^{*}(k)\right)
\end{aligned}
$$

where $\kappa=(1-\beta)-1 / C<0$.

Now we first show forward invariance of $P$. To this end, let $x^{*}(k) \in P$, i.e., $V_{\beta}\left(x^{*}(k)\right) \leq$ $\alpha_{2}(\vartheta) / \beta$ and distinguish two cases.

Case 1: $V_{\beta}\left(x^{*}(k)\right) \leq \alpha_{2}(\vartheta)$. In this case (3.6) and $g\left(x^{*}(k), u^{*}(k)\right) \geq 0$ imply

$$
V_{\beta}\left(x^{*}(k+1)\right) \leq V_{\beta}\left(x^{*}(k)\right) / \beta \leq \alpha_{2}(\vartheta) / \beta
$$

from which $x^{*}(k+1) \in P$ follows.

Case 2: $V_{\beta}\left(x^{*}(k)\right)>\alpha_{2}(\vartheta)$. This condition implies $\left\|x^{*}(k)-x^{e}\right\|>\vartheta$ and, since $P \subset Y$, also $\left\|x^{*}(k)-x^{e}\right\| \leq \Theta$. Hence, (3.8) applies and in particular yields $V_{\beta}\left(x^{*}(k+1)\right) \leq V_{\beta}\left(x^{*}(k)\right) \leq$ $\alpha_{2}(\vartheta) / \beta$, and thus again $x^{*}(k+1) \in P$.

Next we show forward invariance of $Y$. Hence, let $x^{*}(k) \in Y$, i.e., $V_{\beta}(x) \leq \alpha_{1}(\Theta)$, which implies $\left\|x^{*}(k)-x^{e}\right\| \leq \Theta$. If $x^{*}(k) \in P$ then forward invariance of $P$ implies $x^{*}(k+1) \in$ $P \subset Y$. If $x^{*}(k) \notin P$, then $\vartheta \leq\left\|x^{*}(k)-x^{e}\right\|$ holds and from (3.8) we obtain

$$
V_{\beta}\left(x^{*}(k+1)\right)-V_{\beta}\left(x^{*}(k)\right) \leq \frac{\kappa}{\beta} V_{\beta}\left(x^{*}(k)\right)<0,
$$

implying $V_{\beta}\left(x^{*}(k+1)\right) \leq V_{\beta}\left(x^{*}(k)\right) \leq \alpha_{1}(\Theta)$ and thus $x^{*}(k+1) \in Y$.

Finally, we now show the existence of $\eta \in \mathcal{K} \mathcal{L}$ such that the inequality (2.7) holds for all optimal trajectories starting in $Y$ as long as they are outside $P$. For all $x^{*}(k) \in Y \backslash P$, $\vartheta \leq\left\|x^{*}(k)-x^{e}\right\| \leq \Theta$ is satisfied, thus inequality (3.8) applies and yields

$$
V_{\beta}\left(x^{*}(k+1)\right) \leq \sigma V_{\beta}\left(x^{*}(k)\right)
$$

for $\sigma=\kappa / \beta+1=(C-1) /(C \beta)$. Since $C \geq 1$ we obtain $\sigma \in[0,1)$. Hence, $V_{\beta}\left(x^{*}(k)\right) \leq$ $\sigma^{k} V_{\beta}\left(x^{*}(0)\right)$ decreases exponentially. From this and from (3.5) we obtain

$$
\left\|x^{*}(k)-x^{e}\right\| \leq \alpha_{1}^{-1}\left(V_{\beta}\left(x^{*}(k)\right)\right) \leq \alpha_{1}^{-1}\left(\sigma^{k} V_{\beta}\left(x^{*}(0)\right)\right) \leq \alpha_{1}^{-1}\left(\sigma^{k} \alpha_{2}\left(\left\|x^{*}(0)-x^{e}\right\|\right)\right)
$$

as long as $x^{*}(k) \notin P$, which proves the claim since $\eta(r, k)=\alpha_{1}^{-1}\left(\sigma^{k} \alpha_{2}(r)\right)$ is a $\mathcal{K} \mathcal{L}$ function. 
Remark 3.2: (i) The proof shows that the optimal value function $V_{\beta}$ is a Lyapunov function in the sense of, e.g., [15, Definition 2.18].

(ii) The inequality $V_{\beta}(x) \leq \alpha_{2}\left(\left\|x-x^{e}\right\|\right)$ follows from (3.3) for $\alpha_{2}=C \gamma \operatorname{if} \inf _{u \in \mathbb{U}} g(x, u) \leq$ $\gamma\left(\left\|x-x^{e}\right\|\right)$ holds for some $\gamma \in \mathcal{K}_{\infty}$ and all $x \in \mathbb{X}$. Typical choices of $g$ penalizing the distance from an equilibrium, such as $g(x, u)=\left\|x-x^{e}\right\|^{\kappa}+\lambda\left\|u-u^{e}\right\|^{\kappa}$ satisfy this inequality for any $\lambda \geq 0, \kappa>0$.

(iii) Since $g$ in Theorem 3.1 is nonnegative, the inequality $V_{\beta} \leq V_{1}$ holds for all $\beta \in(0,1]$. Hence, if there exists $C>0$ such that (3.3) holds for $\beta=1$ (which is similar to a condition used in model predictive control, see, e.g., [33, 16] and [15, Remark 6.15]), then (3.3), (3.4) hold for all $\beta$ sufficiently close to 1 .

\section{Strictly dissipative problems}

The requirement that $g$ is positive definite is fairly strong and it was observed in [27] that it can be replaced by a weaker detectability condition. Here we use an alternative concept to detectability, namely strict dissipativity.

Definition 4.1: (i) Given a discount factor $0<\beta<1$, we say that the system $(2.2)$ is discounted strictly dissipative with supply rate $s: \mathbb{Y} \rightarrow \mathbb{R}$ at an equilibrium $\left(x^{e}, u^{e}\right)$ if there exists a storage function $\lambda: \mathbb{X} \rightarrow \mathbb{R}$ bounded from below with $\lambda\left(x^{e}\right)=0$ and a class $\mathcal{K}_{\infty}$-function $\alpha$ such that the inequality

$$
s(x, u)+\lambda(x)-\beta \lambda(f(x, u)) \geq \alpha\left(\left\|x-x^{e}\right\|\right)
$$

holds for all $(x, u) \in \mathbb{Y}$ with $f(x, u) \in \mathbb{X}$.

(ii) We say that the optimal control problem (2.1) is discounted strictly dissipative at an equilibrium $\left(x^{e}, u^{e}\right)$ if the system $(2.2)$ is discounted strictly dissipative at $\left(x^{e}, u^{e}\right)$ with supply rate $s(x, u)=g(x, u)-g\left(x^{e}, u^{e}\right)$.

The following proposition (Proposition 3 in [12]) relates this concept to the optimal control problem we study in this paper.

Proposition 4.2: Consider the discounted optimal control problem (2.1) with discount factor $0<\beta<1$ and assume the problem is discounted strictly dissipative with bounded storage function $\lambda$. Then the optimal trajectories of (2.1) coincide with those of the problem

$$
\min _{u \in \mathcal{U}} \widetilde{J}_{\beta}\left(x_{0}, u\right) \text { with } \widetilde{J}_{\beta}\left(x_{0}, u\right):=\sum_{k=0}^{\infty} \beta^{k} \tilde{g}_{\beta}(x(k), u(k))
$$

with stage cost

$$
\tilde{g}_{\beta}(x, u)=g(x, u)-g\left(x^{e}, u^{e}\right)+\lambda(x)-\beta \lambda(f(x, u))
$$

which is positive definite w.r.t. $x^{e}$ at $\left(x^{e}, u^{e}\right)$.

We recall that positive definiteness of the stage cost was defined in Theorem 3.1 and we note that for positive definite $g$ strict dissipativity is always satisfied with $\lambda \equiv 0$. 
The proof of this proposition in [12] moreover shows that $J_{\beta}$ and $\widetilde{J}_{\beta}$ are related via

$$
\widetilde{J}_{\beta}(x, u)=J_{\beta}(x, u)-\frac{g\left(x^{e}, u^{e}\right)}{1-\beta}+\lambda(x) .
$$

Analogously to $V_{\beta}$ we define the optimal value function of the modified problem as

$$
\widetilde{V}_{\beta}\left(x_{0}\right):=\inf _{u(\cdot) \in \mathcal{U}_{a d}} \widetilde{J}_{\beta}\left(x_{0}, u(\cdot)\right) .
$$

The following corollary is then immediate from Theorem 3.1.

Corollary 4.3: Consider a strictly dissipative discounted optimal control problem in the sense of Definition 4.1(ii) at an equilibrium $\left(x^{e}, u^{e}\right) \in \mathbb{X} \times \mathbb{U}$. Assume that the optimal value function $\widetilde{V}_{\beta}$ of the modified problem satisfies $\widetilde{V}_{\beta}(x) \leq \alpha_{2}\left(\left\|x-x^{e}\right\|\right)$ and

$$
\widetilde{V}_{\beta}(x) \leq C \inf _{u \in \mathbb{U}} \tilde{g}_{\beta}(x, u)
$$

for all $x \in \mathbb{X}$ with $\vartheta \leq\left\|x-x^{e}\right\| \leq \Theta$ for $0 \leq \vartheta<\Theta$, a function $\alpha_{2} \in \mathcal{K}_{\infty}$, and a constant $C \geq 1$ satisfying

$$
C<1 /(1-\beta)
$$

Then, whenever $\alpha_{1}(\Theta)>\alpha_{2}(\vartheta) / \beta$, there are forward invariant sets $P \subset Y \subset \mathbb{X}$ with $P \subset$ $\bar{B}_{\delta}\left(x^{e}\right), \delta=\alpha_{1}^{-1}\left(\alpha_{2}(\vartheta) / \beta\right), Y \supset B_{\Delta}\left(x^{e}\right) \cap \mathbb{X}, \Delta=\alpha_{2}^{-1}\left(\alpha_{1}(\Theta)\right)$, such that the equilibrium $\left(x^{e}, u^{e}\right)$ is $P$-practically asymptotically stable on $Y$ for the optimally controlled system. Particularly, the optimal closed-loop system is $(\delta, \Delta)$-practically asymptotically stable. If (4.4) holds for all $x \in \mathbb{X}$ then, the equilibrium is asymptotically stable for the optimally controlled system.

Proof. By Proposition 4.2 we know that $\tilde{g}_{\beta}$ is positive definite. Hence, the assumptions of the corollary imply all assumptions of Theorem 3 for the modified problem and the assertion follows since again by Proposition 4.2 the optimal trajectories of the problems coincide.

A potential problem with Corollary 4.3 is that the main limiting condition (4.4) is formulated in terms of the function $\widetilde{V}_{\beta}$ which is in general difficult to compute. However, we can use this corollary in order to derive the following theorem which does not need a condition of this type.

Theorem 4.4: Assume there is $0<\beta_{0}<1$ such that for all $\beta \in\left(\beta_{0}, 1\right)$ the optimal control problem (2.1) is strictly dissipative at an equilibrium $\left(x_{\beta}^{e}, u_{\beta}^{e}\right)$ with bounded storage functions $\lambda=\lambda_{\beta}$, possibly depending on $\beta$, and $\alpha \in \mathcal{K}_{\infty}$ in (4.1) independent of $\beta$. Assume there are $\alpha_{\lambda}, \alpha_{V} \in \mathcal{K}_{\infty}$ such that the inequalities

$$
\lambda_{\beta}(x) \leq \alpha_{\lambda}\left(\left\|x-x^{e}\right\|\right) \quad \text { and } \quad V_{\beta}(x)-\frac{g\left(x^{e}, u^{e}\right)}{1-\beta} \leq \alpha_{V}\left(\left\|x-x^{e}\right\|\right)
$$

hold for all $x \in \mathbb{X}$ and $\beta \in\left(\beta_{0}, 1\right)$.

Then for each $\Delta>\delta>0$, there is $\beta^{*} \in\left(\beta_{0}, 1\right)$ and $\eta \in \mathcal{K} \mathcal{L}$ such that the closed-loop is $(\delta, \Delta)$-practically asymptotically stable for each $\beta \in\left(\beta^{*}, 1\right)$. 
Proof. The inequalities on $\lambda_{\beta}$ and $V_{\beta}$ from (4.6) together with (4.3) imply that

$$
\widetilde{V}_{\beta}(x) \leq \alpha_{2}\left(\left\|x-x^{e}\right\|\right)
$$

for all $\beta \in\left(\beta_{0}, 1\right)$ with $\alpha_{2}=\alpha_{\lambda}+\alpha_{V}$. Moreover, $\tilde{g}_{\beta}(x, u) \geq \alpha_{1}\left(\left\|x-x^{e}\right\|\right)$ follows for all these $\beta$ with $\alpha_{1}=\alpha$ from (4.1). Given $\Delta>\delta>0$, we define $\vartheta=\alpha_{2}^{-1}\left(\beta \alpha_{1}(\delta)\right)$ and $\Theta=\alpha_{1}^{-1}\left(\alpha_{2}(\Delta)\right.$, which ensures that $P_{\beta}=P$ and $Y_{\beta}=Y$ from (3.7) satisfy $P_{\beta} \subset \bar{B}_{\delta}\left(x^{e}\right)$ and $Y_{\beta} \supset B_{\Delta}\left(x^{e}\right)$. Since we consider varying $\beta \in\left(\beta_{0}, 1\right)$, we explicitly indicate the possible dependence of these sets on $\beta$. Let

$$
C:=\sup _{\substack{\beta \in\left(\beta_{0}, 1\right) \\ \vartheta \leq\left\|x-x^{e}\right\| \leq \Theta}} \frac{\widetilde{V}_{\beta}(x)}{\inf _{u \in \mathbb{U}} \tilde{g}_{\beta}(x, u)},
$$

which is finite because of the upper bound $\alpha_{2}$ on $\widetilde{V}_{\beta}$ and the lower bound $\alpha_{1}$ on $\tilde{g}_{\beta}$. Then, (4.4) holds and by chosing $\beta^{*} \in(1-1 / C, 1)$, for all $\beta \in\left(\beta^{*}, 1\right)$ inequality (4.5) is satisfied, too. Hence, all assumptions of Corollary 4.3 hold for all these $\beta$ and thus the desired $(\delta, \Delta)$-practical asymptotic stability follows.

The property proved in Theorem 4.4 is also called semiglobal practical asymptotic stability w.r.t. $\beta$, cf., e.g., Siljak [31, p. 312] or Isidori [20, p. 126].

Example 4.5: Consider the scalar optimal control problem with dynamics and stage cost function

$$
x(k+1)=2 x(k)+u(k), \quad g(x, u)=u^{2}
$$

and state and control constraints $\mathbb{X}=[-1,1], \mathbb{U}=[-2,2]$. It is straightforward to check that the optimal control problem is strictly dissipative with storage function $\lambda(x)=-\frac{3}{2} x^{2}$ for all $\beta \in(0.4,1)$. We note that in this example $\lambda$ is independent of $\beta$. Using the control $u(0)=-2 x, u(1)=u(2)=\ldots=0$ one also easily sees that $V_{\beta}(x) \leq g(x, u(0))=4 x^{2}$. Hence, (4.6) holds and we can conclude semiglobal practical asymptotic stability for $\beta<1$ sufficiently close to 1 .

For the unconstrained problem, an optimal feedback law can be computed via the associated Riccati equation using the rescaled dynamics $x(k+1)=\sqrt{\beta} 2 x(k)+\sqrt{\beta} u(k)$, cf. the discussion preceding Example 6.5, below. This Riccati equation exhibits two solutions, the optimal feedback law $\mu^{*}(x) \equiv 0$ and the optimal stabilizing feedback law $\mu^{*}(x)=-\frac{4 \beta-1}{2 \beta} x$. Among these two feedback laws, only the second one is a candidate for an optimal feedback law respecting the constraints, because the closed-loop for $u(k)=\mu^{*}(x(k)) \equiv 0$ obviously violates the state constraints for all initial conditions $x_{0} \neq 0$. For the second feedback law, the resulting closed-loop system is

$$
x(k+1)=\frac{1}{2 \beta} x(k)
$$

and thus one sees that for $\beta \in(0.5,1)$ the optimal discounted feedback law is stabilizing for the original dynamics (4.7) and maintains the constraints. Hence, in this example we obtain asymptotic stability, not merely practical asymptotic stability, with discounted optimal control. Remark 6.4(i) provides an explanation for this phenomenon. Note that the speed of convergence towards 0 slows down as $\beta$ becomes smaller. 
Remark 4.6: Under appropriate regularity conditions, it is shown in [24, Theorem 5] that strict dissipativity with respect to $x$ and $u$ of the undiscounted problem, i.e., Definition 4.1 with $\beta=1$ and $\alpha\left(\left\|x-x^{e}\right\|+\left\|u-u^{e}\right\|\right)$, implies strict dissipativity of the discounted problem for $\beta<1$ and $\beta$ sufficiently close to 1 . The construction of the respective functions in the proof of [24, Theorem 5] shows that the uniform bounds (4.6) on $\lambda_{\beta}$ and $V_{\beta}$ for $\beta \in\left(\beta_{0}, 1\right)$ hold. Hence, the assumptions of Theorem 4.4 follow from strict dissipativity w.r.t. $x$ and $u$ of the undiscounted problem.

Remark 4.7: We note that in all statements the distance $\left\|x-x^{e}\right\|$ could be replaced by a more general function $\sigma: \mathbb{X} \rightarrow \mathbb{R}_{\geq 0}$. This allows to extend the results to the stabilization of a set $A:=\{x \in \mathbb{X} \mid \sigma(x)=0\}$ instead of the equilibrium $x^{e}$.

\section{$5 \quad$ Strict dissipativity and detectability}

Remark 4.6 reveals an analogy between Theorem 4.4 and the results in [27], in which an (undiscounted) detectability condition, first considered in [8], is used in place of the strict dissipativity condition from Definition 4.1(ii).

In [27], the inequalities ${ }^{2}$

$$
g(x, u) \geq 0, \quad V_{\beta}(x) \leq \alpha_{2}\left(\left\|x-x^{e}\right\|\right) \quad \text { for all } \beta \in(0,1),
$$

and the following definition were assumed to hold.

Definition 5.1: The optimal control problem (2.1) is called detectable at an equilibrium $\left(x^{e}, u^{e}\right)$, if there exists a continuous function $W: \mathbb{X} \rightarrow \mathbb{R}_{\geq 0}, \alpha_{W}, \chi_{W} \in \mathcal{K}_{\infty}$ and $\bar{\alpha}_{W} \in \mathcal{N}^{3}$ such that for all $(x, u) \in \mathbb{X} \times \mathbb{U}$ the inequalities

$$
\begin{aligned}
W(x) & \leq \bar{\alpha}_{W}\left(\left\|x-x^{e}\right\|\right) \\
W(f(x, u))-W(x) & \leq-\alpha_{W}\left(\left\|x-x^{e}\right\|\right)+\chi_{W}(g(x, u))
\end{aligned}
$$

hold.

Assuming the inequalities (5.1) and Definition 5.1, a semiglobal practical stability statement similar to our Theorem 4.4 was shown in [27].

It is known that there are examples which are strictly dissipative but do not satisfy these conditions. In fact, Example 4.5 is one of these examples, as it was verified in [10] that it does not satisfy the detectability condition. Another example is the following.

Example 5.2: Consider the nonlinear Van de Vusse chemical reactor model from [23, 28], given by the equations

$$
\begin{aligned}
\dot{c}_{A} & =r_{A}\left(c_{A}, \vartheta\right)+\left(c_{i n}-c_{A}\right) u_{1} \\
\dot{c}_{B} & =r_{B}\left(c_{A}, c_{B}, \vartheta\right)-c_{B} u_{1} \\
\dot{\vartheta} & =h\left(c_{A}, c_{B}, \vartheta\right)+\alpha\left(u_{2}-\vartheta\right)+\left(\vartheta_{i n}-\vartheta\right) u_{1},
\end{aligned}
$$

\footnotetext{
${ }^{2}$ In [27] the more general setting using $\sigma$ from Remark 4.7 is employed. Here we use $\left\|x-x^{e}\right\|$, instead, in order to keep the presentation technically simple.

${ }^{3} \alpha: \mathbb{R}_{0}^{+} \rightarrow \mathbb{R}_{0}^{+}$is an $\mathcal{N}$-function if it is continuous and non decreasing with $\alpha(0)=0$.
} 
where the state variables $c_{A}$ and $c_{B}$ are the concentrations of species $A$ and $B$, respectively, in mol/l, $\vartheta$ is the reactor temperature in ${ }^{\circ} \mathrm{C}$, and the auxiliary functions are given by

$$
\begin{aligned}
r_{A}\left(c_{A}, \vartheta\right)= & -k_{1}(\vartheta) c_{A}-2 k_{3}(\vartheta) c_{A}^{2} \\
r_{B}\left(c_{A}, c_{B}, \vartheta\right)= & k_{1}(\vartheta) c_{A}-k_{2}(\vartheta) c_{B} \\
h\left(c_{A}, c_{B}, \vartheta\right)= & -\delta\left(k_{1}(\vartheta) c_{A} \Delta H_{A B}+k_{2}(\vartheta) c_{B} \Delta H_{B C}\right. \\
& \left.\quad+2 k_{3}(\vartheta) c_{A}^{2} \Delta H_{A D}\right) \\
k_{i}(\vartheta)= & k_{i 0} \exp \frac{-E_{i}}{\vartheta+\vartheta_{0}}, \quad i=1,2,3 .
\end{aligned}
$$

The system parameters can be found in [28, Table 1]. The inputs $u_{1}, u_{2}$ are the normalized flow rate of $A$ through the reactor in $1 / \mathrm{h}$ and the temperature in the cooling jacket in ${ }^{\circ} \mathrm{C}$. The states and inputs are subject to the constraints

$$
\begin{array}{lll}
c_{A} \in[0,6] \frac{\mathrm{mol}}{l} & c_{B} \in[0,4] \frac{\mathrm{mol}}{l} & \vartheta \in[70,200]^{\circ} \mathrm{C} \\
u_{1} \in[3,35] \frac{1}{h} & u_{2} \in[0,200]^{\circ} \mathrm{C} . &
\end{array}
$$

Here the optimization objective is to maximize the output of the species $B$ in the reactor, which results in the running cost

$$
g(x, u)=-x_{2} u_{1}
$$

A discrete time model was obtained by sampling the continuous time model with sampling time $T=0.0033$ and zero order hold. For this example it was verified in [5] that (nondiscounted) strict dissipativity holds at $\left(x^{e}, u^{e}\right)=\left((2.175,1.105,128.5)^{T},(35,142.8)^{T}\right)$. It is straightforward to see that strict dissipativity for the continuous time system verified in [5] implies strict dissipativity for the discrete time sampled data model. Hence, the problem fits the framework of this paper, but since the cost function is negative, it does not fit the detectability framework of [27].

The following Figure 5.1 shows the closed-loop behavior for different discount factors, where we denote $x_{1}=c_{A}, x_{2}=c_{B}$ and $x_{3}=\vartheta \cdot 10^{-2}$ (the rescaling of $x_{3}$ was made in order to improve the numerical stability, see also [4, Section 3.3]). The optimal solutions were computed numerically via a receding horizon approach with optimization horizon $N=50$ using ACADO [19]. For $\beta=0.999$ the solutions are visually indistinguishable from their undiscounted counterparts, see [4, Section 3.3] and converge to an equilibrium with a distance from $x^{e}$ of order $10^{-3}$. For smaller $\beta$, the deviation from the equilibrium $x^{e}$ caused by the merely practical stability is clearly visible.

Examples 4.5 and 5.2 show that the strict dissipativity based result from Theorem 4.4 covers optimal control problems which are not detectable. In general, we can make the following statement about the relation between detectability and strict dissipativity for the undiscounted problem, i.e., Definition 4.1 for $\beta=1$.

Proposition 5.3: Consider an optimal control problem which satisfies (5.1) and is detectable in the sense of Definition 5.1 with $\chi_{W}(r) \leq C r$ for some $C>0$ and all $r \geq 0$. Then the undiscounted problem is strictly dissipative. 



Figure 5.1: Optimal solutions for the van de Vusse reactor for different discount factors $\beta$

Proof. Multiplying all involved functions by $1 / C$ we may assume $C=1$. The second inequality in (5.1) yields $V_{\beta}\left(x^{e}\right)=0$, which implies that for $x(0)=x^{e}$ there is $u(\cdot) \in \mathcal{U}_{a d}$ with $g(x(k), u(k))=0$ for all $k \in \mathbb{N}_{0}$. Since $W\left(x^{e}\right)=0$ and $W \geq 0$, the second inequality in Definition 5.1 implies $W\left(f\left(x^{e}, u(0)\right)\right)=0$. Again from the second inequality in Definition 5.1 we then obtain $\alpha_{W}\left(\left\|f(x, u)-x^{e}\right\|\right) \leq \chi_{W}\left(g\left(x^{e}, u(0)\right)\right)=0$ which implies $f\left(x^{e}, u(0)\right)=$ $x^{e}$. This implies that $u(0)$ is an equilibrium control value, which we denote by $u^{e}$, for which $g\left(x^{e}, u^{e}\right)=0$ holds. Using the existence of this pair $\left(x^{e}, u^{e}\right)$, by [13, Proposition 3.3] it is sufficient to show that there is $\alpha \in \mathcal{K}_{\infty}$ such that

$$
\sup _{K \geq 0, u \in \mathbb{U}^{K}} \sum_{k=0}^{K-1}-\left(g(x(k), u(k))-\alpha\left(\left\|x(k)-x^{e}\right\|\right)\right)<\infty .
$$

Choosing $\alpha=\alpha_{W}$, for any $K>0$ and $u \in \mathbb{U}^{K}$ we obtain

$$
\begin{aligned}
& \sum_{k=0}^{K-1}-\left(g(x(k), u(k))-\alpha\left(\left\|x(k)-x^{e}\right\|\right)\right) \\
& \leq \sum_{k=0}^{K-1}-\left(\chi_{W}(g(x(k), u(k)))-\alpha_{W}\left(\left\|x(k)-x^{e}\right\|\right)\right) \\
& \leq \sum_{k=0}^{K-1}\left(W(x(k))-W(x(k+1))=W(x(0))-W(x(K)) \leq W(x(0))=W\left(x_{0}\right),\right.
\end{aligned}
$$

where we used $W(x(K)) \geq 0$. This immediately implies (5.4) and thus the assertion.

This means that at least for special cases of detectability, strict dissipativity is a weaker assumption. Whether this statement can be extended to the case of general $\chi_{W}$ is currently an open problem.

\section{$6 \quad$ Alternative conditions}

In this section we present two alternative conditions which guarantee non-practical asymptotic stability. The first is a controllability type condition which ensures the inequalities 
(3.3), (3.4) hold for all $x \in \mathbb{X}$, the second is a relaxation of these inequalities. A concluding example illustrates the conservativeness of our conditions.

Controllability conditions for ensuring bounds on optimal value functions are well known in the context of model predictive control, see [9] or [15, Assumption 6.4]. They allow the computation of an upper bound on the value function. The condition that fits our setting is the following.

Definition 6.1: Let $\left(x^{e}, u^{e}\right) \in \mathbb{X} \times \mathbb{U}$ be an equilibrium with $g\left(x^{e}, u^{e}\right)=0$. We say that the system is asymptotically controllable to $x^{e}$ with respect to the cost $g$, if there are $K>0$ and $\sigma \in(0,1)$ such that for each initial condition $x_{0} \in \mathbb{X}$ there exists an admissible control $u \in \mathcal{U}_{a d}$ with

$$
g(x(k), u(k)) \leq K \sigma^{k} \inf _{u \in \mathbb{U}} g\left(x_{0}, u\right)
$$

for all $k \geq 0$.

Remark 6.2: We note that this definition is satisfied, e.g., for costs of the form $g(x, u)=$ $\left\|x-x^{e}\right\|^{\kappa}, \kappa>0$, if the system is exponentially controllable to $x^{e}$. This means that there are $L>0$ and $\omega \in(0,1)$ such that for each $x_{0}$ there is an admissible control with $\left\|x(k)-x^{e}\right\| \leq L\left\|x_{0}-x^{e}\right\| \omega^{k}$. In this case, one easily computes that Definition 6.1 holds with $K=L^{\kappa}$ and $\sigma=\omega^{\kappa}$.

Proposition 6.3: Assume that the condition from Definition 6.1 is satisfied. Then (3.3) holds with $C=\frac{K}{1-\beta \sigma}$ for all $x \in \mathbb{X}$.

Proof. Fix $x_{0} \in \mathbb{X}$ and let $u \in \mathcal{U}_{a d}$ be the control from Definition 6.1. Then we have

$$
V_{\beta}\left(x_{0}\right) \leq \sum_{k=0}^{\infty} \beta^{k} g(x(k), u(k)) \leq K \inf _{u \in \mathbb{U}} g\left(x_{0}, u\right) \sum_{k=0}^{\infty}(\beta \sigma)^{k}=\frac{K}{1-\beta \sigma} \inf _{u \in \mathbb{U}} g\left(x_{0}, u\right)
$$

which proves the claim.

Remark 6.4: (i) From Proposition 6.3 and Theorem 3.1 one easily concludes non-practical asymptotic stability for $\beta<1$ and $\beta$ sufficiently close to 1 , because for such $\beta$ condition (3.4) is satisfied. This also explains the non-practical asymptotic stability of the closedloop in Example 4.5, because in this example it is easily checked that the condition from Definition 6.1 is satisfied for the modified stage cost $\tilde{g}$.

(ii) In the situation of Remark 6.2, Proposition 6.3 implies that the assumptions of Theorem 3.1 are satisfied if the inequality $C<1 /(1-\beta)$ holds for $C=K /(1-\beta \sigma), \sigma=\omega^{\kappa}$ and $K=L^{\kappa}$. This is equivalent to $\beta$ satisfying the inequality

$$
\beta \geq \frac{L^{\kappa}-1}{L^{\kappa}-\omega^{\kappa}}
$$

This inequality is always satisfied for $\beta$ sufficiently close to 1 , because the right hand side of (6.1) is less than 1 since $\omega^{\kappa}<1$. Note also that the expression on the right is decreasing for decreasing $\kappa$, hence choosing a smaller $\kappa$ yields a larger range of discount factors $\beta$ for which asymptotic stability can be ensured by Theorem 3.1.

Proposition 6.3 shows that the upper bound (3.3) on $V_{\beta}$ imposed in Theorem 3.1 can be ensured without knowing an optimal feedback law. However, this can clearly be conservative. Our second alternative condition reduces this conservatism. To this end, we observe 
that the first part of the proof and (2.6) reveals that for the optimally controlled system inequality (3.3) can be replaced by

$$
V_{\beta}(x) \leq C g\left(x, \mu^{*}(x)\right),
$$

where $\mu^{*}$ denotes the optimal feedback law, provided it exists. Obviously, the conditions (3.3) and (6.2) only differ if $g$ depends on $u$ in a nontrivial way. In this case, however, condition (6.2) can be significantly less conservative, as the first part of Example 6.5, below, shows.

As in the proof of Theorem 3.1 one sees that the existence of $C<1 /(1-\beta)$ satisfying $(6.2)$ is sufficient for $V_{\beta}$ being a Lyapunov function for the system. Moreover, it is also "almost" necessary for $V_{\beta}$ being a Lyapunov function, because if there is $x \in \mathbb{X}$ with $x \neq x^{e}$ for which (6.2) does not hold for any $C<1 /(1-\beta)$, then $V_{\beta}$ will not strictly decrease in this point and will thus not be a Lyapunov function ${ }^{4}$.

However, the optimal value function being a Lyapunov function is not a necessary condition for the discounted optimally controlled system to be asymptotically stable - not even in the linear quadratic case, as the second part of the following example shows.

Example 6.5 illustrates the conservativeness of the conditions (3.3), (3.4) and (6.2), (3.4). We have intentionally selected a simple linear quadratic example in order to ensure the existence of a linear optimal feedback law such that we can determine asymptotic stability of the closed-loop by computing eigenvalues. An optimal control problem is called linear quadratic if

$$
f(x, u)=A x+B u \quad \text { and } \quad g(x, u)=x^{T} Q x+u^{T} R u
$$

for matrices $A, B, Q$ and $R$ of appropriate dimensions. It is well known that the undiscounted infinite horizon optimal value function for this problem is given by $V_{1}(x)=x^{T} P x$ where $P \in \mathbb{R}^{n \times n}$ solves the discrete time algebraic Riccati equation

$$
P=A^{T} P A-A^{T} P B\left(R+B^{T} P B\right)^{-1} B^{T} P A+Q
$$

and the optimal feedback law is given by

$$
\mu^{*}(x)=\left(R+B^{T} P B\right)^{-1} B^{T} P A x,
$$

see, e.g., [2, Section 9.2.6]. The discounted functional for $\beta \in(0,1)$ can be rewritten as

$$
\sum_{k=0}^{\infty} \beta^{k}\left(x(k)^{T} Q x(k)+u(k)^{T} R u(k)\right)=\sum_{k=0}^{\infty} \hat{x}(k)^{T} Q \hat{x}(k)+\hat{u}(k)^{T} R \hat{u}(k)
$$

with $\hat{x}(k)=\sqrt{\beta^{k}} x(k)$ and $\hat{u}(k)=\sqrt{\beta^{k}} u(k)$. Since $\hat{x}$ and $\hat{u}$ satisfy the equation

$$
\begin{aligned}
\hat{x}(k+1) & =\sqrt{\beta^{k+1}} x(k+1)=\sqrt{\beta^{k+1}}(A x(k)+B u(k)) \\
& =\sqrt{\beta} A \sqrt{\beta^{k}} x(k)+\sqrt{\beta} B \sqrt{\beta^{k}} u(k)=\sqrt{\beta} A \hat{x}(k)+\sqrt{\beta} B \hat{u}(k),
\end{aligned}
$$

\footnotetext{
${ }^{4}$ We note that this condition is only "almost" necessary because it might happen that (6.2) holds with an $x$-dependent constant $C(x)$ which satisfies $C(x)<1 /(1-\beta)$ for all $x \in \mathbb{X}$ but $\sup _{x \in \mathbb{X}} C(x)=1 /(1-\beta)$, which is neither a contradiction to the strict decrease property of a Lyapunov function nor is it sufficient for the proof of Theorem 3.1. However, if we denote the infimal $C$ for which (6.2) holds for all $x \in \mathbb{X}$ and fixed $\beta \in(0,1)$ by $C_{\beta}$ and assume that $\beta \mapsto C_{\beta}-1 /(1-\beta)$ is strictly decreasing, then this exceptional situation can only happen for one single value of $\beta$.
} 
the discounted problem is equivalent to the undiscounted problem with matrices $\sqrt{\beta} A$, $\sqrt{\beta} B, Q$ and $R$. Hence, the linear quadratic infinite horizon discounted optimal control problem can be solved via the discrete time algebraic Riccati equation with matrices $\sqrt{\beta} A$ and $\sqrt{\beta} B .^{5}$ In the example, below, this equation was solved numerically using the DARE routine in MAPLE. All numerical results were rounded to three or four significant digits.

Example 6.5: Consider the linear system $x(k+1)=A x(k)+B u(k)$ with

$$
A=\left(\begin{array}{ll}
2 & 0 \\
1 & 2
\end{array}\right) \text { and } B=\left(\begin{array}{ll}
1 & 0 \\
0 & 1
\end{array}\right) \text {. }
$$

We consider the quadratic stage cost $g(x, u)=x^{T} Q x+u^{T} R u$ with

$$
R=Q=\left(\begin{array}{ll}
1 & 0 \\
0 & 1
\end{array}\right)
$$

We first note that since both $x \mapsto \inf _{u} g(x, u)$ and $V_{\beta}$ are quadratic functions with $V_{\beta}(x)>$ $\inf _{u} g(x, u)$, a $C$ satisfying (3.3), (3.4) exists for $\beta$ sufficiently close to 1 . By computing $V_{\beta}$ via the Riccati equation, one can check numerically that such a $C$ exists if and only if $\beta$ is larger than $\approx 0.846$.

A numerical computation for $\beta=0.4$ yields the optimal value function $V_{\beta}(x)=x^{T} P x$ and the optimal controller $\mu^{*}(x)=-K x$ with

$$
P=\left(\begin{array}{ll}
4.39 & 1.46 \\
1.46 & 3.12
\end{array}\right) \quad \text { and } \quad K=\left(\begin{array}{ll}
1.33 & 0.199 \\
0.728 & 1.06
\end{array}\right)
$$

By maximizing $V_{\beta}(x) / \inf _{u \in \mathbb{U}} g(x, u)$ w.r.t. $x$, one checks that the minimal $C$ satisfying (3.3) for all $x$ evaluates to $C \approx 5.34$, which is considerably larger than $1 /(1-\beta)=1 / 0.6=$ $5 / 3=1 . \overline{6}$. Hence, the criterion from Theorem 3.1 does not hold. However, maximizing $V_{\beta}(x) / g\left(x, \mu^{*}(x)\right)$, one sees that the minimal $C$ satisfying $(6.2)$ for all $x$ equals to $C \approx 1.45$, which is smaller than $1 /(1-\beta)$. Hence $V_{\beta}$ is still a Lyapunov function for the optimally controlled system, even though the criterion in Theorem 3.1 fails to hold. Numerically, this situation persists until $\beta$ decreases to $\approx 0.3342$.

The same computation for $\beta=0.334$, however, yields the optimal value function $V_{\beta}(x)=$ $x^{T} P x$ and the optimal controller $\mu^{*}(x)=-K x$ with

$$
P=\left(\begin{array}{ll}
4.10 & 1.33 \\
1.33 & 2.86
\end{array}\right) \quad \text { and } \quad K=\left(\begin{array}{ll}
1.22 & 0.201 \\
0.667 & 0.932
\end{array}\right)
$$

For $x=(0.109,0.994)^{T}$ one checks that

$$
V_{\beta}(A x-B K x)-V_{\beta}(x)=0.00269>0
$$

implying that $V_{\beta}$ increases along the closed-loop solution and is therefore not a Lyapunov function for the closed-loop system. On the other hand, the eigenvalues of $A-B K$ are $0.924 \pm 0.215 i$ with modulus $0.949<1$, which shows that the closed-loop system is

\footnotetext{
${ }^{5}$ This fact appears to be anecdotally known but we were not able to find a reference in the literature, hence we provided this brief explanation here.
} 
asymptotically stable although $V_{\beta}$ is not a Lyapunov function. This situation holds until $\beta \approx 0.3109$. For smaller values of $\beta$, asymptotic stability of the closed-loop no longer holds.

Summarizing, in this example condition (3.3) is satisfied for $\beta \in[0.846,1]$, condition (6.2) holds for $\beta \in[0.3342,1]$ and the optimal feedback renders the origin asymptotically stable for $\beta \in[0.312,1]$. For $\beta \in(0,0.311]$, asymptotic stability of the origin is lost.

\section{Conclusions}

We provided sufficient conditions for the asymptotic stabilization of an equilibrium point when using optimal controls derived from a discounted optimal control problem. A first result, Theorem 3.1, relies on an appropriate bound on the optimal value function. Based on this result, in Theorem 4.4 we derived a semiglobal practical asymptotic stability result w.r.t. the discount factor $\beta$ for strictly dissipative optimal control problems. Two examples illustrated that this analysis applies to classes of systems for which asymptotic stability cannot be ensured by the detectability based analysis proposed in [27]. Finally, we derived alternative conditions ensuring asymptotic stability, not merely practical asymptotic stability, and illustrated their conservativeness by another example. These results provide key building blocks for demonstrating provable stability properties in economic and social applications such as described in $[7,17,18]$.

\section{References}

[1] D. Angeli, R. Amrit, and J. B. Rawlings. On average performance and stability of economic model predictive control. IEEE Trans. Autom. Control, 57(7):1615-1626, 2012.

[2] P. J. Antsaklis and A. N. Michel. A Linear Systems Primer. Birkhauser, 2007.

[3] D. P. Bertsekas. Dynamic Programming and Optimal Control. Vol. 1 and 2. Athena Scientific, Belmont, MA, 1995.

[4] T. Faulwasser, L. Grüne, and M. A. Müller. Economic nonlinear model predictive control. Preprint, University of Bayreuth, 2017. epub.uni-bayreuth.de/3318/.

[5] T. Faulwasser, M. Korda, C. N. Jones, and D. Bonvin. On turnpike and dissipativity properties of continuous-time optimal control problems. Automatica, 81:297-304, 2017.

[6] V. Gaitsgory, L. Grüne, and N. Thatcher. Stabilization with discounted optimal control. Syst. Contr. Lett., 82:91-98, 2015.

[7] D. Grass, J. P. Caulkins, G. Feichtinger, G. Tragler, and D. A. Behrens. Optimal Control of Nonlinear Processes. With Applications in Drugs, Corruption, and Terror. Springer-Verlag, Berlin, 2008.

[8] G. Grimm, M. J. Messina, S. E. Tuna, and A. R. Teel. Model predictive control: for want of a local control Lyapunov function, all is not lost. IEEE Trans. Automat. Control, 50(5):546-558, 2005. 
[9] L. Grüne. Analysis and design of unconstrained nonlinear MPC schemes for finite and infinite dimensional systems. SIAM J. Control Optim., 48(2):1206-1228, 2009.

[10] L. Grüne. Optimal invariance via receding horizon control. In Proceedings of the 50th IEEE Conference on Decision and Control and European Control Conference - CDC 2011, pages 2668-2673, Orlando, Florida, 2011.

[11] L. Grüne. Approximation properties of receding horizon optimal control. Jahresber. Dtsch. Math.-Ver., 118(1):3-37, 2016.

[12] L. Grüne, C. M. Kellett, and S. R. Weller. On a discounted notion of strict dissipativity. In Proceedings of NOLCOS 2016, volume 49 of IFAC-PapersOnLine, pages 247-252, 2016.

[13] L. Grüne and M. A. Müller. On the relation between strict dissipativity and the turnpike property. Syst. Contr. Lett., 90:45-53, 2016.

[14] L. Grüne and D. Nešić. Optimization based stabilization of sampled-data nonlinear systems via their approximate discrete-time models. SIAM Journal on Control and Optimization, 42(1):98-122, 2003.

[15] L. Grüne and J. Pannek. Nonlinear Model Predictive Control. Theory and Algorithms. Springer-Verlag, London, 2011.

[16] L. Grüne and A. Rantzer. On the infinite horizon performance of receding horizon controllers. IEEE Trans. Automat. Control, 53(9):2100-2111, 2008.

[17] L. Grüne, W. Semmler, and M. Stieler. Using nonlinear model predictive control for dynamic decision problems in economics. Journal of Economic Dynamics \& Control, 60:112-133, 2015.

[18] S. Hafeez, S. R. Weller, and C. M. Kellett. Impact of climate model parametric uncertainty in an MPC implementation of the DICE integrated assessment model. In Proceedings of the 20th IFAC World Congress, Toulouse, France, 2017. To appear.

[19] B. Houska, H. J. Ferreau, and M. Diehl. ACADO toolkit - an open-source framework for automatic control and dynamic optimization. Opt. Control Appl. Methods, 32(3):298-312, 2011.

[20] A. Isidori. Nonlinear control systems. II. Communications and Control Engineering Series. Springer-Verlag London, Ltd., London, 1999.

[21] R. E. Kalman. Contributions to the theory of optimal control. Bol. Soc. Mat. Mexicana, 5:102-119, 1960.

[22] C. M. Kellett and A. R. Teel. Discrete-time asymptotic controllability implies smooth control-Lyapunov function. Systems and Control Letters, 52(5):349-359, August 2004.

[23] K.U. Klatt, S. Engell, A. Kremling, and F. Allgöwer. Testbeispiel: Rührkesselreaktor mit Parallel- und Folgereaktion. In S. Engell, editor, Entwurf nichtlinearer Regelungen, pages 425-432. Oldenbourg-Verlag, 1995. 
[24] M. A. Müller and L. Grüne. On the relation between dissipativity and discounted dissipativity. In Proceedings of the 56th IEEE Conference on Decision and Control CDC 2017, Melbourne, Australia, 2017. To appear.

[25] W. Nordhaus. Estimates of the social cost of carbon: Concepts and results from the DICE-2013R model and alternative approaches. Journal of the Association of Environmental and Resource Economists, 1(1/2):273-312, March 2014.

[26] R. Postoyan, L. Busoniu, D. Nešić, and J. Daafouz. Stability of infinite-horizon optimal control with discounted cost. In Proc. of the 53rd IEEE Conference on Decision and Control, pages 3903-3908, Los Angeles, California, USA, December 2014.

[27] R. Postoyan, L. Busoniu, D. Nešić, and J. Daafouz. Stability analysis of discretetime infinite-horizon optimal control with discounted cost. IEEE Transactions on Automatic Control, 62(6):2736-2749, 2017.

[28] R. Rothfuß, J. Rudolph, and M. Zeitz. Flatness based control of a nonlinear chemical reactor model. Automatica, 32(10):1433-1439, 1996.

[29] J. Rust. Dynamic programming. In S. N. Durlauf and L. E. Blume, editors, The New Palgrave Dictionary of Economics. Palgrave Macmillan, second edition, 2008.

[30] A. Seierstad and K. Sydsæter. Optimal Control Theory with Economic Applications. North-Holland, Amsterdam, 1987.

[31] D. Siljak. Nonlinear Systems: The Parameter Analysis and Design. John Wiley and Sons, 1969.

[32] E. D. Sontag. A Lyapunov-like characterization of asymptotic controllability. SIAM J. Control and Optimization, 21(3):462-471, 1983.

[33] S. E. Tuna, M. J. Messina, and A. R. Teel. Shorter horizons for model predictive control. In Proceedings of the 2006 American Control Conference, pages 863-868, Minneapolis, Minnesota, USA, 2006. 